\title{
$1 \quad$ Effect of imputation on gene network 2 reconstruction from single-cell RNA-seq data
}

$4{ }^{1}$ Department of Computational Molecular Biology, Max Planck Institute for Molecular

5 Genetics, D-14195 Berlin, Germany.

\section{Abstract}

7 Despite the advances in single-cell transcriptomics the reconstruction of gene regulatory

8 networks remains challenging. Both the large amount of zero counts in experimental data

9 and the lack of a consensus preprocessing pipeline for single-cell RNA-seq data make it

10 hard to infer networks from transcriptome data. Data imputation can be applied in order to

11 enhance gene-gene correlations and facilitate downstream data analysis. However, it is

12 unclear what consequences imputation methods have on the reconstruction of gene

13 regulatory networks.

14 To study this question, we evaluate the effect of imputation methods on the performance and

15 structure of the reconstructed networks in different experimental single-cell RNA-seq data

16 sets. We use state-of-the-art algorithms for both imputation and network reconstruction and

17 evaluate the difference in results before and after imputation. We observe an inflation of

18 gene-gene correlations that affects the predicted network structures and may decrease the

19 performance of network reconstruction in general. Yet, within the modest limits of achievable

20 results, we also make a recommendation as to an advisable combination of algorithms, while

21 warning against the indiscriminate use of imputation before network reconstruction in 22 general. 


\section{Introduction}

24 Single-cell transcriptomics has revolutionized genomics. In particular, this new type of data is

25 widely assumed to advance the unraveling of regulatory interactions in the cell. Thus, there

26 is great interest in the computational reconstruction of gene regulatory networks (GRNs)

27 from single-cell transcriptome data.

28 Available methods for GRN reconstruction from single-cell RNA-seq (scRNAseq) data draw

29 on a plethora of statistical approaches (1-6)). Pratapa et. al. (6) provide an extensive

30 benchmark study evaluating the performance of various methods. However, for GRN

31 reconstruction several authors have remarked that preprocessing the data is important,

32 mostly due to the sparse nature of the data $(7,8)$. Several computational analysis pipelines

33 have been suggested and are in wide use $(9,10)$. Typically, as one of the early steps, such a

34 pipeline will include a data normalization and/or imputation step, which statistically estimates

35 unobserved read counts in cases where the method deems that experimental or technical

36 noise has led to the absence of a count, i.e., a so-called dropout. While normalization

37 attempts to correct for different read depths between cells $(11,12)$, imputation attempts to

38 recover gene counts by predicting missing data and eventually smoothen gene expression

39 values (13-22). In some tools a prior normalization step is not required but integrated within

40 the imputation method $(20,21)$. Hou et. al (23) extensively evaluated the impact of imputation

41 on clustering, differential expression analysis and pseudotime inference and invoked

42 cautious interpretations of the results.

43 It still remains unclear though how imputation methods affect network structures (24). On the

44 one hand, it is recommended to use imputation to enhance gene regulatory correlations prior

45 to network inference $(18,20)$. But on the other hand, results based on imputed data should 
46 be interpreted with care $(10,23,25)$. Thus, imputation meets conflicting attitudes within the

47 community.

48 Here, we systematically study the question whether data imputation as a preprocessing step

49 affects results obtained using reconstructed GRNs. We build on previously published

50 benchmark studies and consider the best-performing scRNAseq-based tools for both

51 imputation and network reconstruction in our analysis. We measure the performance of

52 different combinations of imputation method and GRN reconstruction method using multiple

53 experimental datasets and a ground truth network that has been used in other benchmark

54 studies. We compare the performance and network structures obtained using unimputed

55 data and imputed data, respectively, and show that in most cases GRN reconstruction does

56 not profit from imputation. In order to explain the observed results we analyze the effect of

57 imputation on predicted gene interactions. Ultimately, we present a recommendation, how to

58 proceed in a data analysis project.

\section{Results}

\subsection{Systematic evaluation of network models}

61 Evaluating the combination between imputation and network inference on different datasets

62 results in a cubic matrix. To manage this we restrict our selection to state-of-the-art

63 computational tools, both for imputation and network inference, that perform most accurately

64 and have been recommended in recent benchmark studies $(6,23)$. Consequently, we

65 developed a computational pipeline to study seven cell types that were obtained from

66 different scRNAseq experiments, using four state-of-the-art imputation methods combined

67 with three top performing GRN methods as depicted in Figure 1. 
68 Information on the seven cell types was derived from five experimental scRNAseq datasets:

69 human embryonic stem cell (hESC) (14), human hepatocytes (hHep) (26), mouse embryonic

70 stem cell (mESC) (27), mouse dendritic cells (mDC) (28) and mouse hematopoietic stem

71 cells (mHSC) (29) that were further separated into the following subtypes: erythrocytes

72 (mHSC-E), granulo monocytes (mHSC-GM) and lymphocytes (mHSC-L).

73 For the four imputation methods, we chose the following methods: two smoothing-based

74 tools magic (18) and knn-smoothing (22); a model-based tool saver (17) and a deep-learning

75 based tool $d c a$ (20). We included $d c a$ because the authors specifically expect to improve

76 network reconstruction. A baseline model was established using normalized but unimputed

77 data.

78 As for GRN reconstruction, we selected three tools: an information-based tool PIDC (4), and

79 two tree-based tools GENIE3 (30) and GRNBoost2 (31). In the remainder of this paper we

80 use the term "model" to refer to the combination of a GRN reconstruction algorithm with an

81 imputation method or no imputation, respectively. We obtain the ground truth network from

82 the STRING database - a functional protein-protein interaction network (32) and use the

83 evaluation framework BEELINE (6) for measuring the performance of each network model

84 (see Methods). Furthermore, we inspect the reconstructed network and compare the results

85 with one another. 

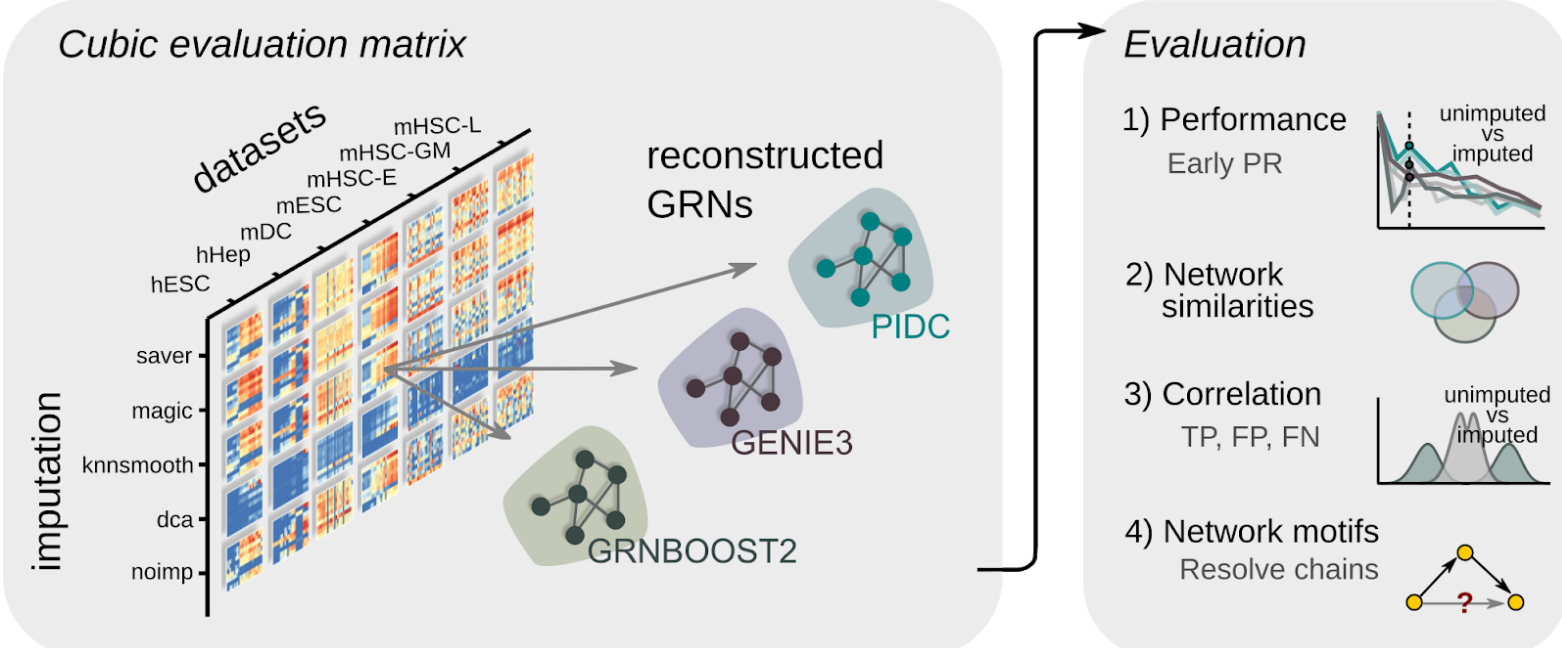

86 Figure 1 | Systematic evaluation of network reconstruction from imputed and unimputed data.

87 Cubic evaluation matrix consists of seven cell types from experimental scRNAseq data, four

88 imputation methods (see text) and three network reconstruction algorithms. Imputed and unimputed

89 ("noimp" in the Figure) scRNAseq data provide input expression matrices which are used by the gene

90 regulatory network (GRN) reconstruction algorithms using the BEELINE framework (6). We evaluate

91 the performances using the early precision ratios (EPR) and compare network results across different

92 models. Additionally, we inspect the effect of gene-gene correlation on prediction classes (true

93 positives (TP), false positives (FP), false negatives (FN)) before and after imputation, and we search

94 for common motifs within the reconstructed networks. hESC: human embryonic stem cells, hHep:

95 human hepatocytes, mDC: mouse dendritic cells, mESC: mouse embryonic stem cells, mHSC-E,

96 mHSC-GM, mHSC-L: mouse hematopoietic stem cells - erythrocytes, granulo monocytes,

97 lymphocytes.

\subsection{Imputation does not improve the performance of network}

\section{9 reconstruction in general}

100 A compact overview of the results obtained under all the models is provided in Figure 2,

101 where each box summarizes results for one GRN reconstruction method. The performance

102 measurements achieved by the respective model on the seven data sets are arranged on a 
103 vertical axis. Two performance measures have been computed: the early precision ratios

104 (EPR) (6) which are shown in the three boxes of Fig. $2 \mathrm{~A}$, and the $\log _{2}$-ratios between

$105 \mathrm{epr}_{\text {imputed }}$ and $\mathrm{epr}_{\text {unimputed }}$ which are shown in the three boxes of Fig. 2B. EPR refers to the

106 number of true positive interactions within the top-k network normalized by the network

107 density. Here, $k$ refers to the number of positive interactions found in the ground-truth

108 network (see Methods). An EPR of 1 indicates a random predictor. The second performance

109 measure compares the performance of an imputation method relative to the performance of

110 not using imputation. Here, a value of zero means no change, while a negative value

111 indicates a detrimental effect of the imputation.

112 The EPR scores for unimputed data that were reported by Pratapa et. al. (6) could be

113 reproduced in our analysis and are illustrated as a dashed line in Figure 2A. Results vary

114 strongly with the datasets; the scores range from approximately 2 (for the mDC dataset) to

1158 (for mHSC-GM), with less variation across GRN reconstruction algorithms. Applying

116 imputation with either dca, knnsmooth or magic, does not improve the performance in any of

117 the GRN reconstruction methods. While in $\mathrm{mDC}$ data the performance scores in each model

118 scatter around the unimputed model, in mHSC-GM data the performance scores vary

119 strongly, dropping from 8 to just below 5 for the magic/GENIE3 model.

120 Focussing on the change of performance due to imputation as measured using the

121 log2-ratios between imputed and unimputed EPR scores, we observe that only saver is able

122 to improve the performance (Fig. 2B). The saver/PIDC model achieves log-fold-ratios up to

$123+0.5$ in 5 out of 7 datasets and 2 out of 7 datasets combined with GENIE3 or GRNBoost2.

124 All other imputation methods worsen the performance with log-fold-ratios down to -1 which

125 represents a performance decline of $100 \%$ in comparison to the unimputed model. 
126 We further asked the question whether data quality as given by sequencing depth is a

127 determinant of the success of imputation prior to GRN reconstruction. To answer this, we

128 simulated cells in silico by downsampling the gene counts of the given experiments to $60 \%$

129 of their sequencing depth, thereby lowering the detection rate (Supp. Fig. 1). The hope

130 would be that imputation has a more beneficial effect in these simulated data sets as

131 compared to the original, higher quality data. However, similar results as above were

132 obtained when we subjected the lower quality in silico data to our analysis pipeline (Supp.

133 Fig. 2). Like with the original datasets, saver/PIDC obtain the highest improvements

134 compared to the downsampled unimputed datasets. Nonetheless on downsampled data,

135 dca, knnsmooth and magic are able to improve performance in some of the tested datasets,

136 although not consistently.

137 Overall, our results demonstrate that our model performances are highly dataset-dependent.

138 Applying imputation on the original data resulted mostly in a drop of performance of GRN

139 reconstruction compared to the unimputed model, although potentially improving

140 performance on low-quality data tested in silico. 
A
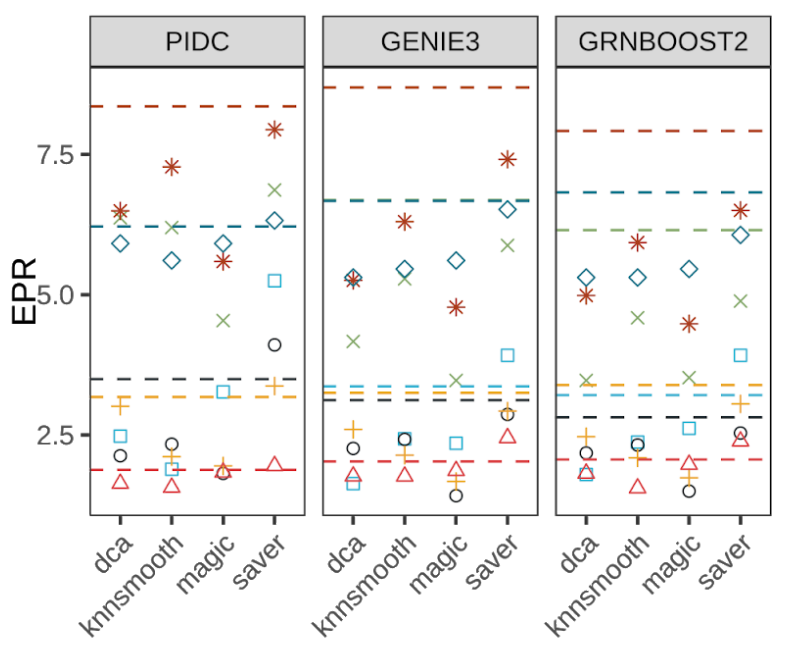

imputation methods
B

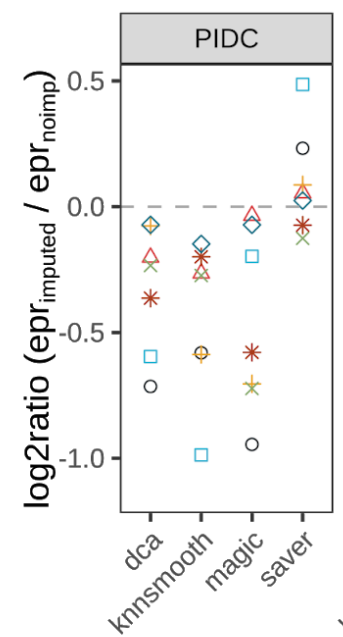

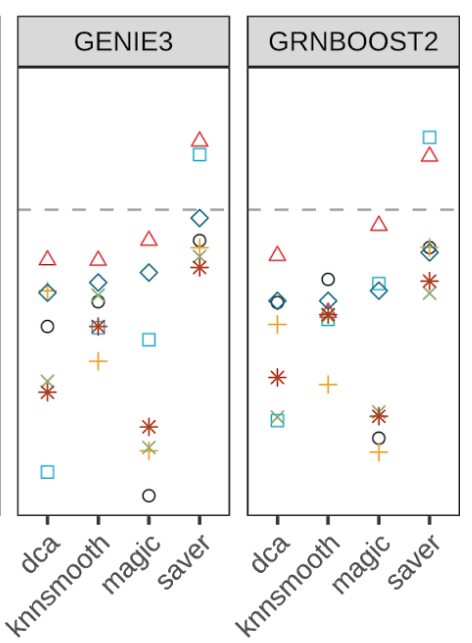

imputation methods

dataset

$\square$ hESC $O$ hHep $\triangle$ mDC

mESC

mHSC-E

* mHSC-GM

$\diamond \mathrm{mHSC}-\mathrm{L}$

141 Figure 2 | Impact of imputation on network reconstruction performances. (A) Absolute EPR scores across

142 imputation methods ( $x$ axis label) and GRN inference algorithms (box) on seven different cell types (coded by

143 shape and color). Dashed lines represent EPR scores obtained without imputation. EPR $=1$ corresponds to a

144 random predictor. (B) log2-ratios between EPR scores obtained using imputed and unimputed data. Log2-ratio =

1450 represents no change in performance (grey dashed line) after imputation.

\subsection{Imputation method rather than GRN method determines}

148 The analysis presented in the preceding Section raises the question how strongly either the

149 choice of imputation method or of network reconstruction algorithm affects the results. To

150 answer this question we first address the variability in results when varying either the one or

151 the other, and then study similarity among computed networks across the models.

152 With regard to the performance variability, we compare the variance of EPR log-fold-ratios

153 under a fixed GRN reconstruction algorithm while varying across imputation methods, and,

154 vice versa, varying the GRN algorithm while keeping the imputation method fixed. As Figure 
155 3A shows EPR log-fold-ratios vary much more strongly when the GRN reconstruction

156 algorithm is fixed than than the other way round (wilcoxon-test $p$-value $\sim 7.86 \times 10^{-6}$ ). This

157 implies that the choice of imputation method determines the quality of results to a larger

158 degree than the choice of GRN reconstruction algorithm.

159 A direct consequence of this observation is the suspicion that the topology of the predicted

160 networks may also be largely determined by the imputation method and to a lesser degree

161 by the GRN reconstruction method. To test this, we inspect the overlap among the 500 most

162 important gene-gene interactions of the computed networks. Here, we calculate pairwise

163 similarity scores using the Jaccard index and use it to hierarchically cluster the networks. We

164 found that networks tend to cluster with respect to imputation methods but not GRN methods

165 (Fig. 3B, Supp. Fig. 4). To make this more precise, we use as a measure of cluster purity the

166 adjusted rand index (ARI) $(33,34)$. ARI coefficients calculated across the seven different cell

167 types show higher cluster purity when labelled with imputation method as opposed to

168 network reconstruction algorithms (Fig. 3C).

169 We conclude that the imputation method largely determines model performance, leaving little

170 influence to the subsequent GRN reconstruction algorithm. The choice of imputation method

171 further biases the outcoming network leading to little consensus across the most important

172 recovered gene-gene interactions as computed based on different imputation methods. 
A

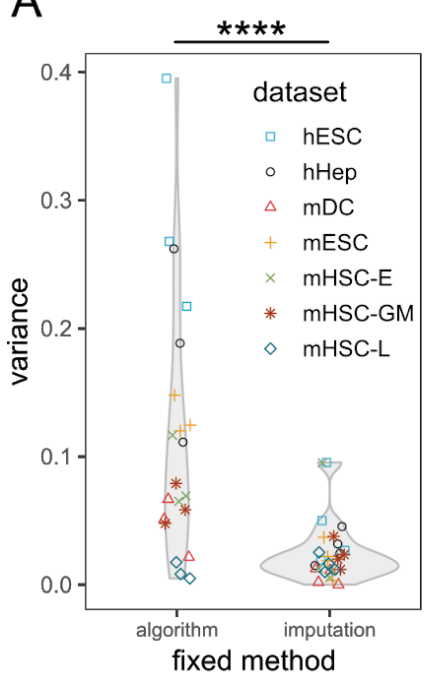

B

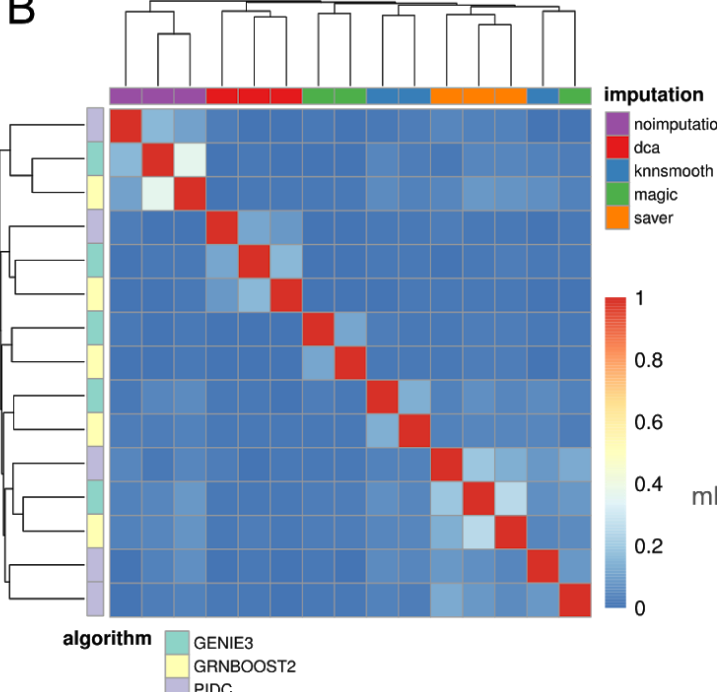

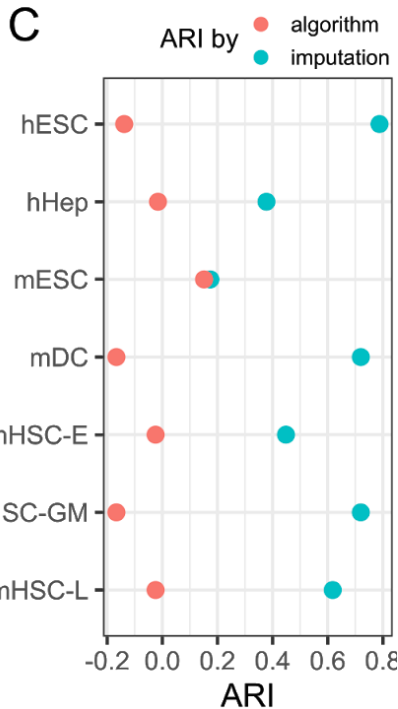

ARI

173 Figure 3 | Variability in network results largely stems from imputation methods. (A) Variance distribution of

174 EPR scores across imputation methods. Left violin plot keeps the GRN algorithm fixed and depicts the variances

175 in EPR log-fold-ratios for each dataset across the imputation methods. Right violin plot shows the variances for

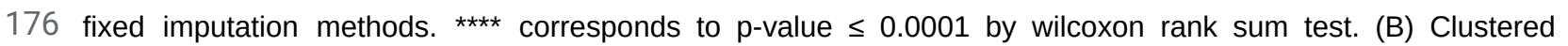

177 heatmap of network similarities measured by Jaccard index within top 500 reported interactions. Columns are

178 color-coded by imputation methods. Rows are color-coded by network inference algorithms. (C) Adjusted rand

179 index (ARI) obtained for clustering results in each cell type by annotation label "algorithm" (pink) and "imputation"

180 (blue), respectively.

\subsection{Inflation of gene-gene correlations and its impact on the}

\section{2 network topology}

183 Based on the reported results, we examine how imputation generally affects gene-gene

184 correlation coefficients. Although not all network reconstruction algorithms use

185 correlation-based measures to recover interactions, we still use Pearson's correlation

186 coefficient as a proxy for the association between two genes. Subsequently, we will

187 investigate whether the interactions within the reconstructed networks affect the global

188 network structure. 
189 Exploring the overall distributions of gene-gene correlations after imputation on scRNAseq

190 data we observe a strong increase in gene-gene correlations (Fig. 4A). Generally,

191 gene-gene correlations go from almost no correlation when computed using unimputed data

192 to very good anti- and positive correlations due to imputation. Here, magic leads to the most

193 extreme enhancement. More specifically, Figure 4B exemplifies the association between

194 three genes before and after imputation, transforming very weak correlations to almost

195 perfect (anti-)correlations. These associations were only reported after imputation using dca

196 among the top-k network using GRNBoost2 in hESC data. Indeed, we commonly find such

197 associations across different datasets and imputation methods.

198 In order to see what impact this enhancement of correlation has on the network structure we

199 next investigated the network density after imputation in relation to the unimputed data using

200 log-ratios (Supp. Fig. 3A). Here, we looked at the top-k networks according to the EPR

201 score. Imputation methods alter the network densities with log-ratios ranging from -0.5 and

$202+0.5$ in hESC, hHep, mDC and mESC data, except for saver and PIDC in hESC data with a

203 slightly higher value of 0.59 . For the three subtypes of mHSC data we observe larger

204 changes in network density reaching log-ratios beyond \pm 1 . Especially here, imputations

205 combined with GENIE3 and GRNBoost2 lead to a sparser network whereas all combinations

206 of imputation methods with PIDC lead to a denser network structure. This is not surprising as

207 GENIE3 and GRNboost2 are network reconstruction algorithms that provide a directed

208 network, whereas PIDC results in undirected interactions providing a backward and forward

209 edge with the same ranks. As we select top-k ranked interactions we take interactions

210 sharing the same ranks simultaneously, consequently leading to a denser network with

211 PIDC. 
212 Besides network density, the network topology is also determined by the node degree

213 distribution. Before imputation we observe a heavy tail node degree distribution

214 predominantly in GENIE3 and GRNBoost2 indicating the presence of many hub nodes

215 (Supp. Fig. 3B). After imputation the heavy tail disappears when using dca, magic and

216 knnsmooth while it still exists when using saver. Generally, PIDC does not lead to this

217 structural change in node degree distribution.

218 As a conclusion, the enhancement of gene-gene correlations due to imputation appears to 219 lead to notable changes in the topology of the predicted gene networks.

\subsection{Increased correlation values in false positive interactions}

\section{1 inflate network results}

222 Since we have observed that imputation may decrease the performance of GRN network

223 reconstruction, we attempt to understand how the altered correlations in imputed data affect

224 network reconstruction. To this end, we explore the change of edge ranks and correlation

225 values of the reported (i.e., positively predicted) and missed (i.e., negatively predicted)

226 interactions.

227 Overall, the ranks of true positive (TP) interactions reported in the unimputed data change

228 significantly after imputation (Fig. 4C, Supp. Tab. 1, Supp. Fig. 5). Some of the previously

229 reported TP interactions could be recovered after imputation. Nevertheless, the majority of 230 previously reported TP interactions shift after imputation towards the end of the gene 231 interaction ranking list, and are considered less important. As a consequence, other 232 interactions become more important.

233 Therefore, we look at the change of correlation of positively predicted interactions before and 234 after imputation. Figure 4D (and Supp. Fig. 6) show scatter plots of gene-gene interactions 
235 with the absolute values of correlation coefficients before imputation on the horizontal axis

236 and the correlation coefficient after imputation on the vertical axis. For each model, red dots

237 are the true positive interactions, yellow are the false positives, and blue are the false

238 negatives. The general shape of the scatter plot reiterates the observation that correlation

239 coefficients tend to get enhanced by imputation. For each class we computed regression

240 lines. For better recognition of true positives after imputation, one would hope for the TP

241 regression line (shown in red in Fig. 4D) to lie well above the others - which is not really the

242 case. We generally observe a strong enhancement of correlations as indicated by the height

243 of the intercept of the regression lines. In 11 out of 12 cases the regression lines for both

244 true and false positive predictions are almost congruent with each other. Note that the red

245 color dominates the other ones and the dots below a red one are not visible.

246 Interestingly, we see remarkably different regression lines if we take the false negative (FN,

247 blue) interactions into account. The majority of FN correlations remain low after imputation,

248 as indicated by the height of the intercept in Fig. 4D. Presumably, the FN correlation values

249 that actually get enhanced get lost in the background due to the inflation of FP correlations in

250 the inferred top-k network. Thus, the boost of correlation values makes it harder for GRN

251 reconstruction methods to separate the actual signal from the background.

252 Many GRN reconstruction methods have the goal of distinguishing direct interactions from

253 transitively inferred ones (35). Therefore, we tested whether the GRN reconstruction

254 algorithms analyzed in this study are able to make the necessary distinction. Given three

255 genes $X, Y$, and $Z$ where $X$ is correlated with $Y$, and $Y$ is correlated with $Z$, these genes form

256 a network chain. However, oftentimes by transitivity these associations seem to imply a

257 correlation between $X$ and Z, thus forming a network loop. Generally, in network theory it is

258 challenging to distinguish chains from loops. In this context, we analyze how the models deal 
259 with the identification of network chains from imputed data. Errors are counted if a

260 supposedly false loop is detected or a chain ist detected instead of a loop (Fig. 4E).

261 In general, PIDC identifies the highest number of network motifs independent of the

262 imputation used. Using saver in combination with PIDC one is able to find the highest

263 number of TP chains. However, saver/PIDC mistakenly identifies network motifs at the same

264 time. In order to measure the performance between true and false predictions we calculate

265 the true positive rates (TPR) and false discovery rates (FDR) for each network inference and

266 imputation method applied to each dataset (Fig. 4F).

267 The performance of network motif search among the top-k networks does not seem to be

268 affected by imputation. Hence, either imputation methods do not necessarily induce

269 transitive correlations or the network reconstruction methods deal well with transitively

270 induced correlations. 
bioRxiv preprint doi: https://doi.org/10.1101/2021.04.13.439623; this version posted April 14, 2021. The copyright holder for this preprint (which was not certified by peer review) is the author/funder, who has granted bioRxiv a license to display the preprint in perpetuity. It is made available under aCC-BY-NC-ND 4.0 International license.

A
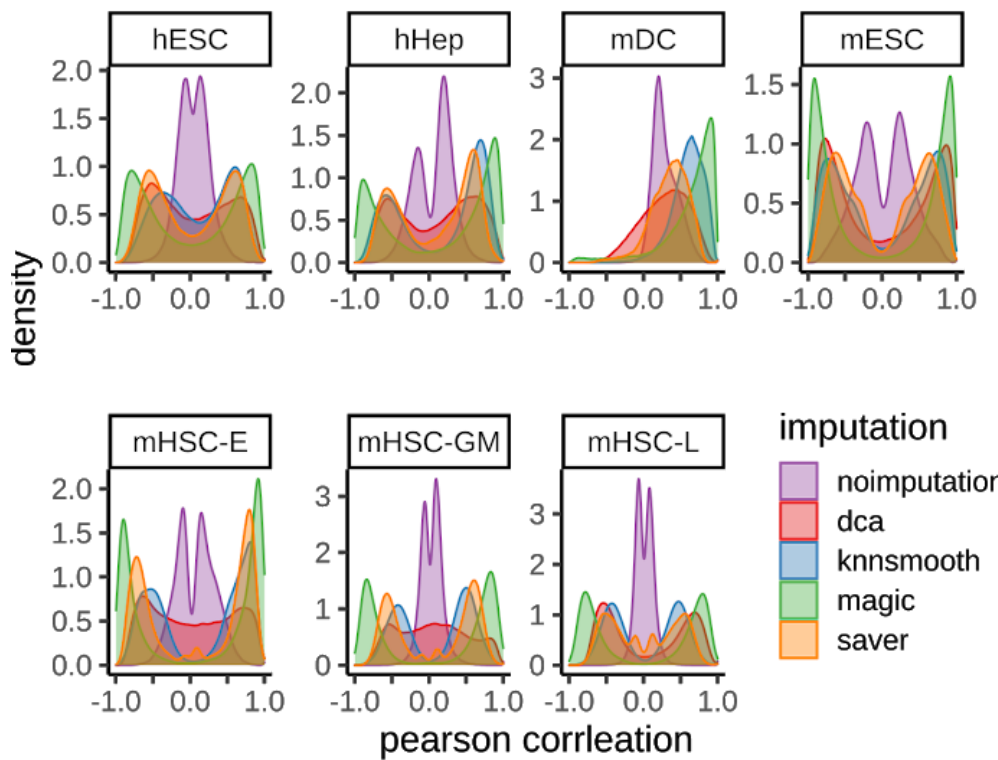

B
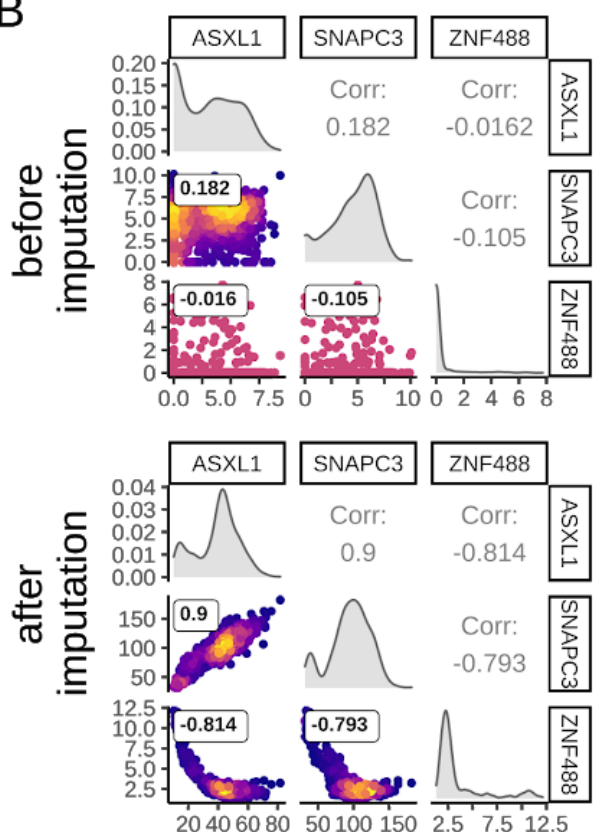

D

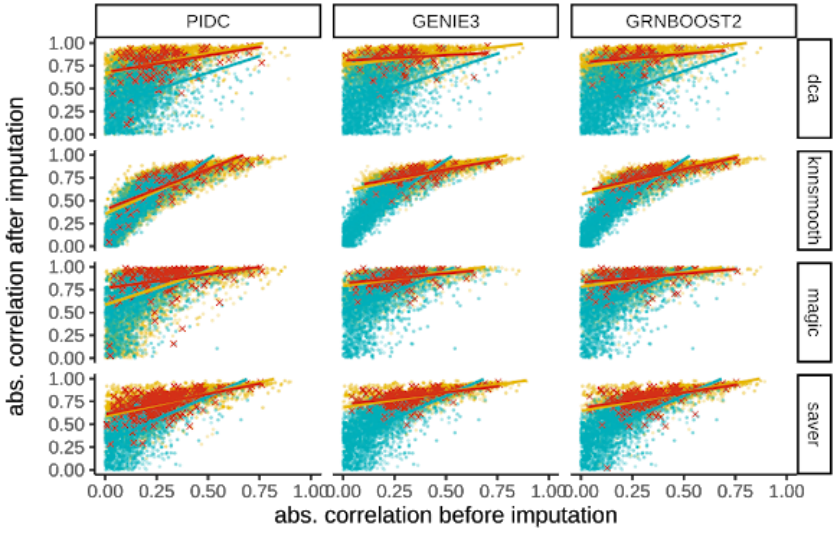

$\mathrm{F}$

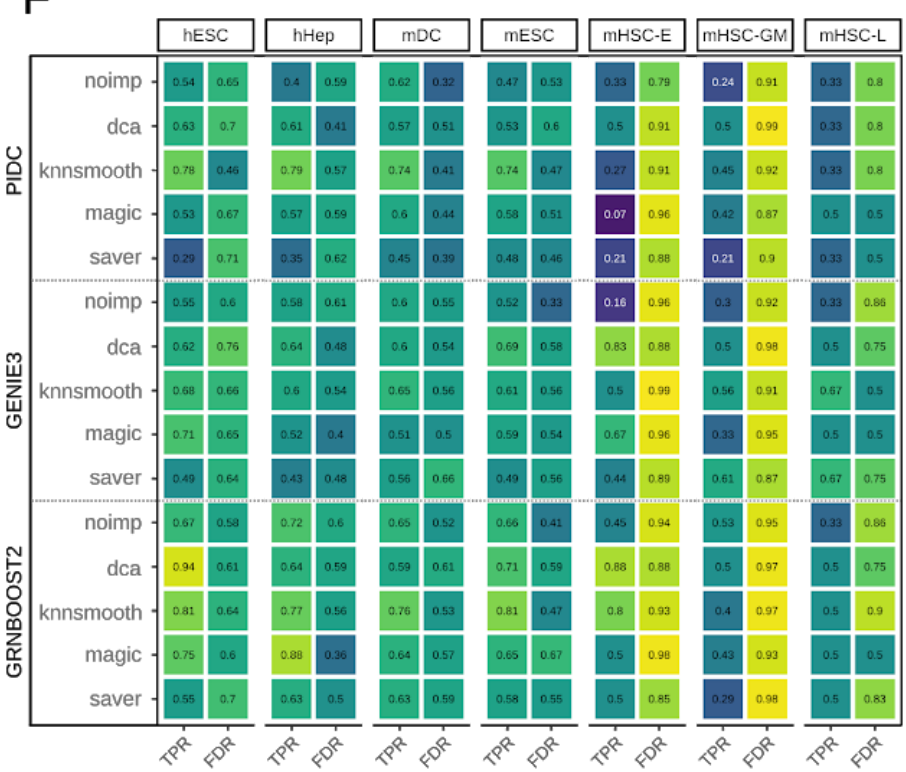

E
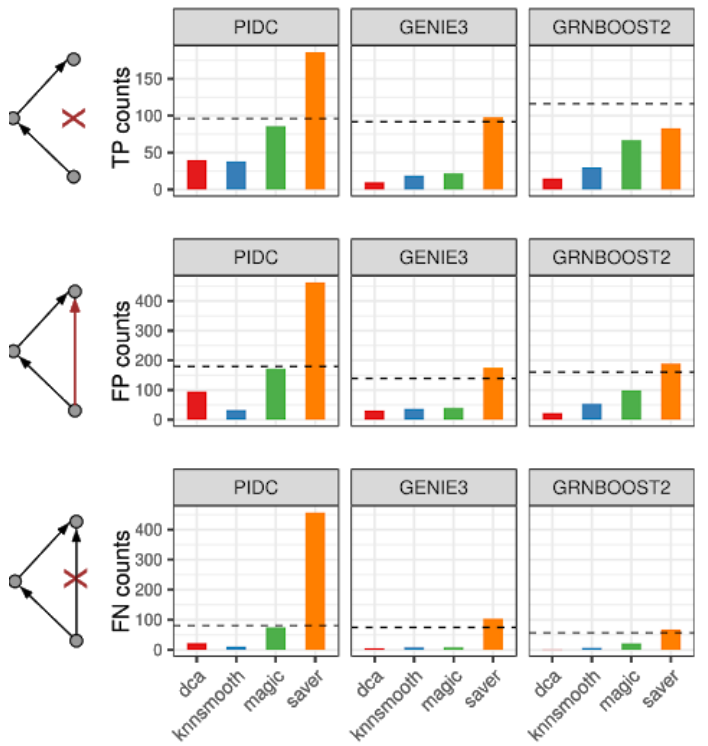

\begin{tabular}{|l|l|l|l|l|l|}
\hline PIDC & GENIE3 \\
\hline
\end{tabular}

ż.
Ranks of true positive edges

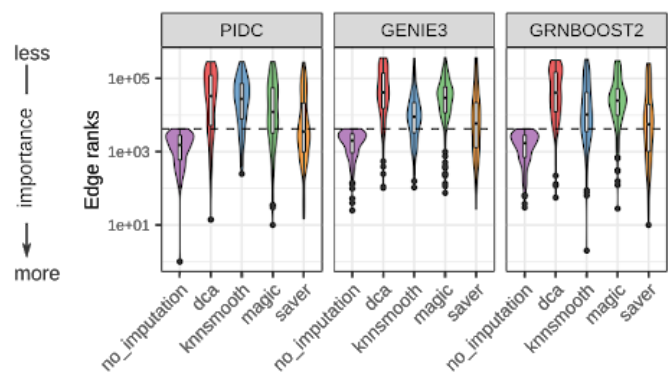

value

0025050075100 


\section{Figure 4 | Gene-gene correlation before and after imputation and its impact on the}

273 predicted interactions. (A) Gene-gene correlation distributions obtained in each cell type

274 color-coded by imputation method among top 500 most variable genes and significantly varying TFs.

275 (B) Paired density scatter plots before and after imputation with dca. GRNBoost2 reported the 276 pairwise interactions between ASXL1, SNAPC3 and ZNF488 among the top-k network after 277 imputation in hESC data. (C) Change of edge ranks in true positive (TP) interactions identified by 278 unimputed model after imputation in hESC data. Dashed line indicates the rank threshold 279 corresponding to the top-k network. Interactions below the dashed line represent TP within the 280 respective model. Low edge ranks represent highly important interactions. (D) Change of correlation 281 values for TP (red crosses), FP (yellow dots) and FN (blue dots) classified by each model in hESC 282 data. Positively predicted interactions differ clearly from FN interactions. (E) Counts of positively and 283 negatively predicted network chain motifs in hESC data for each model. TP network chains agree 284 both in prediction and ground truth. FP network chains are falsely positively predicted chains being 285 actual feed-forward loops in the ground truth. FN network chains are falsely predicted as being 286 feed-forward loops when they are actually network chains in the ground-truth network. (F) TPR and 287 FDR scores for network chain motifs obtained by statistics in E). Ideally, TPR values should be close 288 to 1 whereas FDR values should be close to 0 .

\section{Discussion}

290 The advent of single-cell transcriptomics has rekindled the interest in reconstructing gene

291 regulatory networks from transcriptomics data, primarily for two reasons. Firstly, it is of great

292 interest to study regulation in individual cells in the hope to eventually uncover how, e.g.,

293 differentiation processes proceed. Secondly, the main obstacle in gene network

294 reconstruction from bulk transcriptome data appears to be the low number of available

295 samples in comparison to the large numbers of genes. For example, simulations have 296 demonstrated that high quality reconstruction of gene networks requires a much larger 
297 number of samples than the number of genes (35). Seeing each single cell as a sample, the

298 expectation arose that single-cell transcriptomics would solve this conundrum by providing a

299 sufficiently large number of samples, thus putting high quality network reconstruction within

300 reach.

301 It was sobering for us to see that due to the sparse nature of single-cell RNA-seq data,

302 individual cells cannot contribute as much information to network reconstruction as bulk

303 samples. Indeed, preprocessing of single-cell data for data analysis is crucial (9), and is

304 implemented in many computational pipelines. Imputation has become a possible element of

305 this preprocessing in the hope it would supplement the missing information. In this study we

306 have however demonstrated that the choice of imputation prior to GRN reconstruction

307 influences the results in a two-fold manner: First, it affects the performance of network

308 reconstruction leading to highly variable accuracies and, secondly, the reconstructed

309 network differs significantly between imputation methods.

310 We have systematically evaluated the effect of imputation on GRN reconstruction using

311 experimental scRNAseq data on seven cell types. In this context, we have demonstrated

312 that overall, imputation does not lead to an improvement of GRN reconstruction. However,

313 saver in combination with PIDC may lead in some datasets to an increase in performance.

314 We have shown and thereby agree with previous studies that imputation may boost

315 gene-gene correlations in a dubious way, thereby introducing false positives (25). In turn,

316 these false positives predispose network structures toward forming circular dependencies. In

317 fact, if network reconstruction methods rely on associations that use correlation to some

318 extent (for example regression-based methods) the circularity is highly redundant. Andrews

319 et. al. have warned of this circularity before, albeit in the context of differential expression

320 analysis (36). Consistent with our findings Andrews et. al. showed that saver introduces the 
321 smallest number of spurious gene-gene correlations. We speculate that the combination of

322 saver/PIDC works well because saver is a model-based imputation method and PIDC is a

323 mutual-information based algorithm; the two approaches follow independent assumptions

324 complementing one another, thus avoiding the use of redundant information.

325 In this study we have tested our hypothesis on experimental datasets with fairly large library

326 sizes and gene detection rates (Supp. Fig. 1.). In order to test our hypothesis on more

327 shallowly sequenced single-cell experiments we lowered the detection rate introducing more

328 zero counts in silico. Our results have shown that using saver with PIDC improves results in

329 most cases. However, generally we discourage the indiscriminate usage of imputation prior

330 to GRN reconstruction because imputation tends to introduce a bias into the derived

331 networks. If need be we recommend the use of saver and PIDC. It should be noted that we

332 are not discouraging imputation in general. There may be many other applications that are

333 not studied here, where imputation can be useful, depending on the type of analysis that is

334 subsequently performed.

\section{Methods}

\subsection{Data collection and preprocessing of scRNAseq data:}

337 We collected preprocessed and normalized experimental scRNAseq count data provided in

338 the BEELINE paper (6). Here, the authors also provide the corresponding pseudotime for

339 each dataset / cell type. Please refer to the BEELINE paper for information about

340 preprocessing, normalization, and pseudotime inference.

341 However, dca needs unnormalized raw count data. Therefore, we downloaded the fastq files

342 using the corresponding accession numbers: GSE75748 (hESC) (14), GSE81252 (hHEP) 
343 (26), GSE98664 (mESC) (27), GSE48968 (mDC) (28) and GSE81682 (mHSC) (29). For

344 human and mouse we aligned the fastq files to hg19 (GENCODE release 29) or mm10

345 (GENCODE release M19), respectively and counted the reads per gene using STAR

346 (version 2.7.4a) (37).

347 Following the BEELINE approach, using normalized count data we select the top 500 most

348 variable genes across pseudotime using a general additive model ('gam' R package). In

349 addition to these genes we also include significantly varying TFs (Bonferroni corrected

350 p-value $<0.01)$

351 We filter both imputed and unimputed scRNAseq data using the same set of (i) top 500 most 352 variable genes and (ii) all significantly varying TFs, in order to make a fair comparison 353 between networks inferred using imputed and unimputed data.

\subsection{Code availability}

355 All relevant scripts and $\mathrm{R}$ notebooks for reproducing the results are available at Github

356 (https://github.com/lylamha/imputation_GRN inference). The release includes tutorials from

357 data imputation to the evaluation of the reconstructed networks. It covers the evaluation

358 pipeline with the corresponding analyses and plotting results.

\subsection{Imputation}

360 To impute scRNAseq data we use dca (version 0.2.3), knnsmooth (version 2.1), magic 361 ('Rmagic' $R$ package version 2.0.3) and saver ('SAVER' $R$ package version 1.1.2). Our 362 rationale for selecting knnsmooth, magic and saver is based on a previous comprehensive 363 benchmark evaluation of various imputation methods (23). Additionally, we also include dca 364 as it has been explicitly recommended as improving GRN reconstruction. 
365 We apply each imputation method to normalized count data except for dca where we use the

366 raw counts:

367 dca </path/to/ExpressionData_raw.csv> </path/to/dca_result_folder $>$

368 python3 knn_smooth.py -k 15 -d 10 ।

369 -f <path/to/ExpressionData.csv> ।

370 - o <path/to/ExpressionData_knnsmooth_imputed.csv> --sep ,

371 magic Rsnippet:

372 \# so_dat (seurat object using library(Seurat))

373 so_dat <- magic(so_dat, assay="RNA", genes="all_genes")

374 Dat.magic <- as.data.frame(so_dat@assays\$MAGIC_RNA@data)

375 saver Rsnippet:

376 saver_res <- saver(input_expr_matrix, size.factor $=1$, ncores $=20$,

377 estimates. only $=\mathrm{F}$ )

378 dat.saver <- as.data.frame(saver_res\$estimate)

\subsection{Network reconstruction via BEELINE:}

380 Several tools have been developed to infer GRNs from scRNAseq data differing in their

381 algorithmic approach. They can be categorized into four main classes: correlation-,

382 regression-, mutual information- or modelling-based approaches (6). In this study we

383 evaluated PIDC, GENIE3 and GRNBoost2 which have been previously recommended by

384 Pratapa et. al. (6) . We use the imputed and unimputed scRNAseq data as input matrices

385 for network reconstruction with PIDC, GENIE3 and GRNBoost2 using default parameters. To

386 this end, we use the evaluation framework BEELINE (version 1.0).

387 As part of the BEELINE pipeline we first run 'BLRunner.py' to reconstruct the networks.

388 Then, we filter the reconstructed networks in order to only include interactions from TFs to

389 genes.

390 Finally, we use 'BLevaluater.py' to compute early precision scores evaluating the

391 performance of each network by comparing it to a ground truth network. Here, we choose

392 the functional protein-protein interaction database STRING and filter for genes that only

393 occur in the input expression matrix. 
394 By using early precision scores we only analyze the top-k networks.

\subsection{Characterizing the reconstructed networks}

\section{4.5.1 Top-k network}

397 For comparability reasons we focus our analyses on the top-k networks. The top-k network

398 of a reconstructed network includes the first $k$ interactions selected by their ranks which were

399 assigned by descendingly ordered edge weights. Here, $k$ represents the number of positive

400 interactions in the ground truth network. Interactions can share the same ranks, e.g., the

401 forward and backward interactions in an undirected graph. So with $k$ interactions reported in

402 the ground truth network we select all interactions which ranks are lower than or equal to $k$

403 obtaining the top-k network. Note, that the number of reported interactions can be higher

404 than $k$.

\section{4.5.2 Network density and node degree}

406 Taking into account the interaction between transcription factors and genes only the network

407 density is calculated by numEdges / ((numGenes * numTFs) - numTFs).

408 In order to calculate the node degree we consider all out- and incoming edges for a given

409 node.

\subsection{Methodology of evaluation}

\section{4.6.1 Early Precision Ratios (EPR)}

412 We evaluate the performance of each inferred network based on using early precision sores

413 (EP) which is given by the number of TP divided by the number of positively predicted

414 observations within the top-k network. EP scores were calculated using BEELINE. Each 
415 dataset has a different underlying ground truth subnetwork, hence different evaluations

416 regarding the random predictor. To account for these differences and in order to maintain

417 comparability across datasets we divide the EP scores by the network density (see formula

418 above) of each ground truth subnetwork obtaining EP ratios (EPR). Thus, EPR of 1 is

419 indicative of a random predictor in all experimental datasets. To compare the performance of

420 network inference in each imputation method with the corresponding unimputed data, we

421 calculate log2-ratios between $\mathrm{EPR}_{\text {imputed }}$ and $E \mathrm{PR}_{\text {unimputed }}$.

\section{4.6.2 Network similarities}

423 In order to compare similarities across the reconstructed networks we select the top 500

424 interactions reported in each model. Given two networks, similarity scores are obtained by

425 the Jaccard index which is defined as the number of overlapping interactions divided by the

426 number of unified reported interactions. Repeating this in a pairwise iterative manner we

427 obtain a similarity matrix which we use as an input for a heatmap that is clustered row- and

428 column-wise ('pheatmap' R Package).

429 We calculate adjusted rand index (ARI) scores ('mclust' R package) in order to evaluate the

430 clustering results based on an annotation label (34). As annotation labels we use the

431 network reconstruction algorithm as well as the imputation method. We compare ARI scores

432 across datasets obtained by the two labels using the pairwise wilcoxon rank sum test. 


\section{Acknowledgements}

434 We thank Mahsa Ghanbari and Prabhav Kalaghatgi for comments and proofreading our

435 manuscript, Stefan Börno for assistance with the single-cell RNAseq processing, and

436 Thomas Kreitler for technical support.

\section{Author information}

\section{Affiliation}

439 Max Planck Institute for Molecular Genetics, Berlin, Germany

440 Lam-Ha Ly and Martin Vingron

\section{Contributions}

442 L.H.L. and M.V. designed the study. L.H.L processed and analyzed the data. L.H.L and M.V.

443 wrote the manuscript.

444 Corresponding author

445 Correspondence to Martin Vingron

\section{Competing interests}

447 The authors declare no competing interests. 


\section{Bibliography}

449 1. Ocone A, Haghverdi L, Mueller NS, Theis FJ. Reconstructing gene regulatory dynamics from high-dimensional single-cell snapshot data. Bioinformatics. 2015 Jun 15;31(12):i89-96.

452 2. Specht AT, Li J. LEAP: constructing gene co-expression networks for single-cell RNA-sequencing data using pseudotime ordering. Bioinformatics. 2017 Mar 1;33(5):764-6.

455 3. Aibar S, González-Blas CB, Moerman T, Huynh-Thu VA, Imrichova H, Hulselmans G, et al. SCENIC: single-cell regulatory network inference and clustering. Nat Methods. 2017 Nov;14(11):1083-6.

458 4. Chan TE, Stumpf MPH, Babtie AC. Gene Regulatory Network Inference from Single-Cell Data Using Multivariate Information Measures. Cell Syst. 2017 Sep 27;5(3):251-267.e3.

461 5. Qiu X, Rahimzamani A, Wang L, Ren B, Mao Q, Durham T, et al. Inferring Causal Gene Regulatory Networks from Coupled Single-Cell Expression Dynamics Using Scribe. Cell Syst. 2020 Mar 25;10(3):265-274.e11.

Pratapa A, Jalihal AP, Law JN, Bharadwaj A, Murali TM. Benchmarking algorithms for gene regulatory network inference from single-cell transcriptomic data. Nat Methods. 2020 Jan 6;17(2):147-54.

467 7. Cha J, Lee I. Single-cell network biology for resolving cellular heterogeneity in human diseases. Exp Mol Med. 2020 Nov 26;52(11):1798-808.

469 8. Iacono G, Massoni-Badosa R, Heyn H. Single-cell transcriptomics unveils gene regulatory network plasticity. Genome Biol. 2019 Jun 4;20(1):110.

471 9. Vieth B, Parekh S, Ziegenhain C, Enard W, Hellmann I. A systematic evaluation of single cell RNA-seq analysis pipelines. Nat Commun. 2019 Oct 11;10(1):4667.

10. Luecken MD, Theis FJ. Current best practices in single-cell RNA-seq analysis: a tutorial. Mol Syst Biol. 2019 Jun 19;15(6):e8746.

11. Lun ATL, Bach K, Marioni JC. Pooling across cells to normalize single-cell RNA sequencing data with many zero counts. Genome Biol. 2016 Apr 27;17:75.

477 12. Cole MB, Risso D, Wagner A, DeTomaso D, Ngai J, Purdom E, et al. Performance Assessment and Selection of Normalization Procedures for Single-Cell RNA-Seq. Cell Syst. 2019 Apr 24;8(4):315-328.e8.

480 13. Hou W, Ji Z, Ji H, Hicks SC. A Systematic Evaluation of Single-cell RNA-sequencing Imputation Methods. BioRxiv. 2020 Jan 30;

482 14. Chu L-F, Leng N, Zhang J, Hou Z, Mamott D, Vereide DT, et al. Single-cell RNA-seq reveals novel regulators of human embryonic stem cell differentiation to definitive endoderm. Genome Biol. 2016 Aug 17;17(1):173.

485 15. Lähnemann D, Köster J, Szczurek E, McCarthy DJ, Hicks SC, Robinson MD, et al. 
Eleven grand challenges in single-cell data science. Genome Biol. 2020 Feb 7;21(1):31.

16. Tang W, Bertaux F, Thomas P, Stefanelli C, Saint M, Marguerat S, et al. bayNorm: Bayesian gene expression recovery, imputation and normalization for single-cell RNA-sequencing data. Bioinformatics. 2020 Feb 15;36(4):1174-81.

17. Huang M, Wang J, Torre E, Dueck H, Shaffer S, Bonasio R, et al. SAVER: gene expression recovery for single-cell RNA sequencing. Nat Methods. 2018 Jun 25;15(7):539-42.

18. van Dijk D, Sharma R, Nainys J, Yim K, Kathail P, Carr AJ, et al. Recovering Gene Interactions from Single-Cell Data Using Data Diffusion. Cell. 2018 Jul 26;174(3):716-729.e27.

19. Chen M, Zhou X. VIPER: variability-preserving imputation for accurate gene expression recovery in single-cell RNA sequencing studies. Genome Biol. 2018 Nov 12;19(1):196.

20. Eraslan G, Simon LM, Mircea M, Mueller NS, Theis FJ. Single-cell RNA-seq denoising using a deep count autoencoder. Nat Commun. 2019 Jan 23;10(1):390.

21. Lopez R, Regier J, Cole MB, Jordan MI, Yosef N. Deep generative modeling for single-cell transcriptomics. Nat Methods. 2018 Nov 30;15(12):1053-8.

22. Wagner $F$, Yan $Y$, Yanai I. $K$-nearest neighbor smoothing for high-throughput single-cell RNA-Seq data. BioRxiv. 2017 Nov 21;

23. Hou W, Ji Z, Ji H, Hicks SC. A systematic evaluation of single-cell RNA-sequencing imputation methods. Genome Biol. 2020 Aug 27;21(1):218.

24. Blencowe M, Arneson D, Ding J, Chen Y-W, Saleem Z, Yang X. Network modeling of single-cell omics data: challenges, opportunities, and progresses. Emerg Top Life Sci. 2019 Jul 8;3(4):379-98.

25. Breda J, Zavolan M, van Nimwegen EJ. Bayesian inference of the gene expression states of single cells from scRNA-seq data. BioRxiv. 2019 Dec 29;

26. Camp JG, Sekine K, Gerber T, Loeffler-Wirth H, Binder H, Gac M, et al. Multilineage communication regulates human liver bud development from pluripotency. Nature. 2017 Jun 22;546(7659):533-8.

27. Hayashi T, Ozaki H, Sasagawa $Y$, Umeda M, Danno H, Nikaido I. Single-cell full-length total RNA sequencing uncovers dynamics of recursive splicing and enhancer RNAs. Nat Commun. 2018 Feb 12;9(1):619.

28. Shalek AK, Satija R, Shuga J, Trombetta JJ, Gennert D, Lu D, et al. Single-cell RNA-seq reveals dynamic paracrine control of cellular variation. Nature. 2014 Jun 19;510(7505):363-9.

29. Nestorowa S, Hamey FK, Pijuan Sala B, Diamanti E, Shepherd M, Laurenti E, et al. A single-cell resolution map of mouse hematopoietic stem and progenitor cell differentiation. Blood. 2016 Aug 25;128(8):e20-31.

30. Gao NP, Minhaz Ud-Dean SM, Gunawan R. Gene Regulatory Network Inference 

IFAC-PapersOnLine. 2016;49(26):147-52.

31. Moerman T, Aibar Santos S, Bravo González-Blas C, Simm J, Moreau Y, Aerts J, et al. GRNBoost2 and Arboreto: efficient and scalable inference of gene regulatory networks. Bioinformatics. 2019 Jun 1;35(12):2159-61.

531 32. Szklarczyk D, Gable AL, Lyon D, Junge A, Wyder S, Huerta-Cepas J, et al. STRING v11: protein-protein association networks with increased coverage, supporting functional discovery in genome-wide experimental datasets. Nucleic Acids Res. 2019 Jan 8;47(D1):D607-13.

535 33. Gates AJ, Ahn Y-Y. The impact of random models on clustering similarity. BioRxiv. 2017 Oct 1;

537 34. Hubert L, Arabie P. Comparing partitions. J of Classification. 1985 Dec;2(1):193-218.

538 35. Ghanbari M, Lasserre J, Vingron M. The Distance Precision Matrix: computing networks from non-linear relationships. Bioinformatics. 2019 Mar 15;35(6):1009-17.

540 36. Andrews TS, Hemberg M. False signals induced by single-cell imputation. [version 2; peer review: 4 approved]. F1000Res. 2018 Nov 2;7:1740.

542 37. Dobin A, Davis CA, Schlesinger F, Drenkow J, Zaleski C, Jha S, et al. STAR: ultrafast universal RNA-seq aligner. Bioinformatics. 2013 Jan 1;29(1):15-21. 